\title{
Fundamentos de Informática: exposición y utilidad desde la perspectiva de un alumno
}

\author{
Miguel Hoyo García \\ Grado en Ingeniería de Tecnologías de Telecomunicación. E.T.S de Ing. Informática y de \\ Telecomunicación, Universidad de Granada \\ Granada, España \\ miguelhg@me.com
}

\begin{abstract}
Resumen. Fundamentos de Informática es una asignatura que se imparte en el Grado en Ingeniería de Tecnologías de Telecomunicación durante el primer semestre del primer curso del grado. En este artículo se analizará, desde la perspectiva de un alumno de cuarto curso, la evolución de la asignatura, así como su metodología, las aplicaciones y utilidad de dicha asignatura en años posteriores del grado. Además, se tratarán las fortalezas y debilidades de la asignatura y su método de evaluación.
\end{abstract}

Palabras Clave: Fundamentos de Informática punto vista estudiante .

\begin{abstract}
Fundamentos de Informática (English: Computers Basics) is a subject taught in the Telecomunications' Technologies Engineering Degree during the first semester of the grade. This article will analyse, from a forth year student point of view, the evolution (course) of the subject, as well as its methodology, applications and utility of this subject in the following years of the degree. In addition the strengths and weakness of the subject and the method of evaluation will be exposed.
\end{abstract}

Keywords: Computers Basics point of view student

\section{Introdución}

Fundamentos de Informática se trata de una asignatura básica impartida en el primer semestre del primer curso del Grado en Ingeniería de tecnologías de Telecomunicación.

El temario teórico que se impartió en la asignatura se distribuyó de la siguiente manera:

1. Introducción y conceptos básicos

2. Representación de la información en computadores

3. Estructura funcional de computadores

4. Elementos de programación 
5. Fundamentos de Sistemas Operativos

6. Bases de datos

Por otro lado, el temario práctico fue el siguiente:

1. Uso del sistema Operativo

2. Herramientas informáticas con aplicación en Ingeniería

3. Funcionamiento a bajo nivel de un ordenador

4. Uso básico de un Sistema Gestor de Bases de Datos

Del mismo modo, se impartieron un total de tres seminarios: "Estructura y montaje de un PC", otro seminario sobre el funcionamiento de CODE-2 y por último, un seminario sobre software libre y software de código abierto. Además, se realizaron un total de 11 trabajos adicionales (llamadas en la evaluación como "actividades en casa") relacionados con la materia que se estaba impartiendo en clase.

Por último, el método de evaluación se realizó como se muestra a continuación:

- Evaluación contínua:

Table 1. Método de evaluación contínua.

\begin{tabular}{|c|c|c|c|c|}
\hline $\begin{array}{l}\text { Aspectos a } \\
\text { Evaluar }\end{array}$ & $\begin{array}{l}\text { Técnica de } \\
\text { evaluación }\end{array}$ & Ponderación & Nota máxima & $\begin{array}{l}\text { Nota mínima } \\
\text { para aprobar }\end{array}$ \\
\hline $\begin{array}{l}\text { Seguimiento y } \\
\text { asistencia }\end{array}$ & $\begin{array}{l}\text { Asistencia a } \\
\text { clase de teoría } \\
\text { y actividades } \\
\text { en casa }\end{array}$ & $10 \%$ & 1 & 0 \\
\hline $\begin{array}{l}\text { Prácticas y } \\
\text { seminarios }\end{array}$ & $\begin{array}{l}\text { Evaluación del } \\
\text { trabajo de las } \\
\text { sesiones de } \\
\text { laboratorio y } \\
\text { de los } \\
\text { seminarios }\end{array}$ & $20 \%$ & 2 & 1 \\
\hline \multirow[t]{2}{*}{ Prueba final } & $\begin{array}{l}\text { Examen tipo } \\
\text { test }\end{array}$ & $20 \%$ & 7 & 3,5 \\
\hline & $\begin{array}{l}\text { Examen de } \\
\text { ejercicios } \\
\text { prácticos }\end{array}$ & $50 \%$ & & \\
\hline Total & & $100 \%$ & 10 & 5 \\
\hline
\end{tabular}


- Prueba única final:

Table 2. Método de evaluación con una prueba única final.

\begin{tabular}{|l|c|c|c|}
\hline $\begin{array}{l}\text { Técnica de } \\
\text { evaluación }\end{array}$ & Ponderación & Nota máxima & $\begin{array}{c}\text { Nota mínima } \\
\text { para aprobar }\end{array}$ \\
\hline $\begin{array}{l}\text { Prácticas y } \\
\text { seminarios }\end{array}$ & $20 \%$ & 2 & 1 \\
\hline Examen de Test & $20 \%$ & 2 & 3,5 \\
\hline $\begin{array}{l}\text { Examen de } \\
\text { ejercicios } \\
\text { prácticos }\end{array}$ & $50 \%$ & 5 & \\
\hline Examen teórico & $10 \%$ & 1 & 0 \\
\hline Total & $100 \%$ & 10 & 5 \\
\hline
\end{tabular}

En este artículo se tratará y analizará el desarrollo de la asignatura bajo la experiencia del autor del artículo durante el curso 2010/2011.

\section{Desarrollo de la asignatura}

\subsection{Teoría}

El primer tema se trata de una introducción a conceptos básicos y necesarios para el desarrollo posterior de la asignatura. Conceptos tales como qué son los datos, qué es una codificación, unidades de información (bit y byte), las diferencias entre el soporte físico y el lógico o la estructura funcional de los computadores son estudiados en este primer tema. Además, se explica cómo hacer un análisis de las prestaciones de un computador, es decir, medidas como tasa de transferencia, velocidad del procesador (frecuencia del reloj e instrucciones por segundo), la memoria del sistema, tanto masiva como principal y el tiempo de acceso del computador a los periféricos se analizan en este tema introductorio. Del mismo modo, se detallan la diferencia entre programas e instrucciones y los conceptos lenguaje máquina, lenguaje de alto nivel y el concepto de traductor. Por otro lado, se realiza un estudio de los tipos de computadores existentes, que van desde un servidor hasta un supercomputador. Por último, se analizan los tipos de software de un computador (software de control, sistema operativo y software de aplicaciones), las distintas aplicaciones de la informática y los tipos de aplicaciones de ingeniería con sus distintos campos de aplicación. 
Este primer tema es de gran importancia, pues es la primera toma de contacto con una asignatura sobre informática y se aprenden conceptos que la mayoría estudiábamos por primera vez y que servirán como base de conocimientos de la asignatura en sus posteriores temas.

Una vez se han estudiado los conceptos básicos de la asignatura, se hace un análisis de cómo es representada la información en los computadores en el segundo tema. En primer lugar se estudian los tipos de numeración usuales en informática, tales como el binario, donde se estudiarán sus distintos tipos de representación: coma flotante, complementos a 1, 2, 3... Además de operaciones aritméticas. También se analiza el código hexadecimal y la conversión entre decimal, hexadecimal y binario. Posteriormente, se analiza la representación de textos (SBCD, EBCDIC, ASCII y UNICODE), la representación de sonidos (conversión A/D, códecs...), la representación de imágenes (mapas de bits y sus formatos y mapas de vectores) y por último la representación de datos numéricos. También se estudian métodos de corrección de errores, dónde se introduce el concepto de bit de paridad y finalmente se expone en qué consiste la compresión de datos.

En el tercer tema se realiza un análisis de la estructura funcional de los computadores, es decir, se presenta un análisis de los distintos componentes de un computador, comenzando por las primitivas del nivel de lógica digital (biestables, registros, contadores, ALU, puestos $\mathrm{E} / \mathrm{S}$ y buses) explicando el funcionamiento de cada elemento. Se continúa analizando el procesador y su funcionamiento, estudiando sus elementos internos, cómo se ejecutan las instrucciones y cómo se puede cambiar el orden secuencial de un programa. Seguidamente se analiza el sistema de memoria del computador, donde se diferencia la memoria interna y externa y se introducen conceptos básicos como bloques físicos, tiempo de acceso a memoria, tiempo de ciclo de memoria y ancho de banda. Estos conceptos serán necesarios para la comprensión de la jerarquía de memoria, que consiste en el análisis del rendimiento y la capacidad de los sistemas de memoria mediante tasas de aciertos y fallos, además del funcionamiento de estos sistemas. Así mismo, se explican los conceptos de memoria interna (caché y memoria principal) matizando la diferencia entre ellas y sus funciones además del funcionamiento de cada una, para posteriormente introducir los tipos de memorias externa (soportes magnéticos, discos ópticos y memorias flash USB) explicando cómo funciona cada tipo de memoria, de manera que se puedan comparar los rendimientos de ambos tipos de memorias. Por último se nos presentó cómo se interconecta todo el sistema, para ello se explica qué es un bus y su funcionamiento para finalizar con el concepto de bus del sistema.

El cuarto tema es algo más práctico que los anteriores. En primer lugar se presenta el formato de las instrucciones del lenguaje máquina y su funcionamiento, para después aplicarlo en la práctica con CODE-2. En la segunda parte del tema se profundiza en el funcionamiento teórico de CODE-2, que se complementa con distintos ejemplos prácticos. Finalmente, se explican teóricamente algunos de los lenguajes de alto nivel y el proceso de traducción y ejecución de los programas. 
El quinto, y penúltimo tema, es una introducción a los fundamentos de un sistema operativo. El tema comienza con una definición de "sistema operativo" explicando su objetivos y funciones, además del contexto en el que es utilizado y el método de arranque de un ordenador con un sistema operativo ya instalado. Posteriormente se explica cómo el sistema operativo realiza la gestión de recursos. Primeramente se trata la gestión de recursos del procesador y cómo éste gestiona los procesos desde la monoprogramación hasta los sistemas concurrentes, así mismo se explican los puntos de decisión de la planificación y sus respectivos algoritmos. A continuación se estudia la gestión de memoria por un sistema operativo: la gestión de la memoria principal, incluyendo sus direcciones de memoria y los registros base y se explica en qué consisten las particiones dinámicas y distintos métodos de gestión de la memoria (paginación y segmentación). El tema continúa con el estudio de la gestión de las entradas y salidas, donde se introducen algunos conceptos como la atención de interrupciones y los problemas que se pueden causar si no hay tiempo para tratarlas. Por último, se explica en qué consiste un controlador DMA, así como su funcionamiento y modelos de E/S en sistemas operativos como UNIX y Windows NT. Finalmente, en cuanto a gestión de recursos se refiere, se nos presentó la gestión de archivos, directorios o carpetas, o sea, cómo se organizan y cómo se distribuyen en un sistema operativo. Finalmente se expusieron algunos de los sistemas operativos más relevantes, tales como Windows, UNIX, Linux, Mac OS... Y se exponen algunas de las amenazas a las que éstos están expuestos, además de una categorización de estas amenazas y algunas soluciones a ellas.

Para terminar, en el sexto tema se hace una introducción a las bases de datos explicando en primer lugar conceptos básicos sobre ellas, los tipos de arquitecturas de bases de datos y los modelos de datos (entidades, atributos, relaciones y restricciones) y de bases de datos (distribuidas y orientadas a objetos). Posteriormente se analiza más en profundidad las bases de datos relacionales: su modelo relacional y sus respectivas características. Por último, se presenta cómo es el diseño de una base de datos y sistemas gestores de bases de datos como SQL.

\subsection{Prácticas y seminarios}

Durante el curso se realizaron un total de cuatro prácticas y tres seminarios con objetivos muy diversos: desde elegir los componentes para montar un PC hasta manejar CODE-2 mediante instrucciones máquina.

La primera práctica titulada "Uso del sistema operativo" se trata de una introducción a Linux para que posteriormente lo instalásemos de manera nativa o con máquina virtual a nuestro ordenador. Una vez hecho esto, se pondrían en práctica una serie de órdenes de Linux en la línea de comando y se estudiarían la estructura de directorios de Linux. A continuación se analizaría en entorno gráfico de X-Windows y se pondrían en práctica diferentes comandos para la obtención de información del 
sistema. En último lugar habría que realizar una serie de ejercicios, del que destaca principalmente, la instalación de una aplicación propia de Windows en Linux mediante la herramienta Wine.

En la segunda práctica ("Herramientas informáticas con aplicación en Ingeniería") se centra principalmente en el funcionamiento de MatLab y en la programación de funciones para el mismo MatLab. Una vez explicada la teoría de cómo funcionaba esta herramienta, se realizaron varios ejercicios entre los que hubo que programar algunas funciones, como por ejemplo, una función que calculase la resistencia equivalente a tres resistencias dadas en serie o en paralelo.

Posteriormente se realizó un seminario sobre CODE-2 a modo introductorio, explicando sus instrucciones, su funcionamiento y sus características, para posteriormente, enlazarlo con la práctica tres ("Funcionamiento a bajo nivel de un ordenador") que principalmente trataría sobre CODE-2. En esta práctica nos familiarizaríamos con el entorno de programación de CODE-2, con su repertorio de funciones y entendiendo la función de un programa ensamblador, el cual nos permitiría programar un programa para CODE-2 mediante texto en lugar de hacerlo en código hexadecimal. Por último habría que elegir uno de los ejercicios propuestos que consistían en programar un programa en CODE-2 para posteriormente presentárselo al profesor y corroborar que se ha entendido todo el proceso.

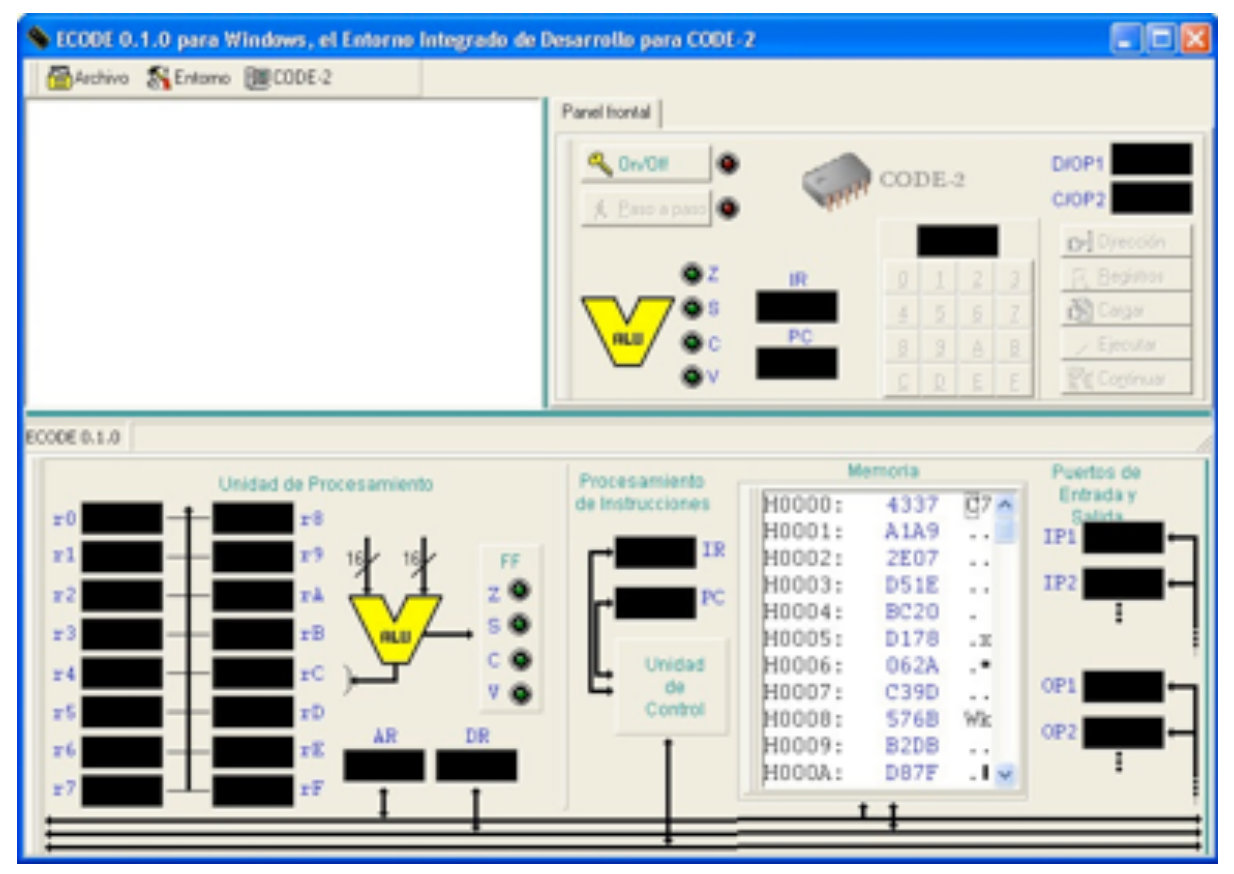

Figura 1. Entorno de ECODE, el simulador de CODE-2. 
En la última práctica (“Uso básico de un Sistema Gestor de Bases de Datos”) hubo que crear una base de datos mediante el programa Microsoft Access. Primero se comenzó con una introducción de cómo crear una base de datos con esta herramienta, cómo realizar consultas a la misma y cómo realizar informes de la base de datos. Una vez hecho esto, había que realizar una serie de seis ejercicios que consistían principalmente en crear una base de datos sobre libros con sus respectivos autores e ir repitiendo los pasos seguidos en la explicación de la práctica. Una vez hechos todos los ejercicios, se presentaría la base de datos al profesor para comprobar su correcto funcionamiento.

Por otro lado, se realizaron dos seminarios más. El primero, "Estructura y montaje de un PC" que consistía en el estudio de los componentes de un ordenador convencional y la interconexión entre sus componentes, para seguidamente realizar algunos ejercicios como: "comprar" nuestro propio PC eligiendo nosotros mismos sus componentes, uno a uno, o realizar un análisis de los componentes de nuestro ordenador.

El último seminario fue sobre software libre y los programas en código abierto. Durante el mencionado seminario se nos explicó los puntos a favor que tienen este tipo de proyectos, además de la historia del software libre y alguno de los proyectos más importantes por aquel momento.

\subsection{Actividades en casa}

Además, se realizaron algunas actividades complementarias a los temas que se estaban dando en la clase de teoría para completar la información proporcionada en el aula. Algunos de estos trabajos fueron: estudio del TOP 500, cómo se codifican algunos símbolos de ASCII, estudio de algunos procesadores INTEL, análisis de las diferencias entre sistemas analógicos y digitales, identificación y análisis de los componentes de nuestro propio ordenador, estudio sobre las amenazas de seguridad (software maligno)...

\section{Perspectiva}

Después de cuatro años estudiando el grado y habiendo cursado todas las asignaturas básicas, troncales y obligatorias de mi rama, he de decir que la asignatura está bastante bien planificada y organizada, facilitando la comprensión de los contenidos y actividades que se realizan en la asignatura.

Además, muchos de los contenidos que se impartieron en la asignatura han servido como base de conocimiento para otras asignaturas del grado, principalmente en asignaturas de electrónica como "Electrónica Digital” donde el uso del código binario es esencial y es impartido por primera vez en esta asignatura. También en la 
asignatura troncal "Sistemas Electrónicos Digitales" los conocimientos impartidos en la asignatura han sido bastante útiles, principalmente para entender del funcionamiento en sí de los sistemas electrónicos y sus respectivas instrucciones, ya que el análisis del funcionamiento del reloj, además de otras características de los sistemas digitales y el estudio y uso de CODE-2 sirvieron como base para esta asignatura, de la misma forma, en la asignatura optativa "Implementación de Algoritmos en Hardware", la introducción a estos sistemas, como CODE-2, ha sido muy útil como punto de partida para la comprensión de los contenidos de esta asignatura.

Por otro lado, la introducción a MatLab durante la práctica dos ha sido para mí esencial a lo largo de todo el grado, ya que en bastantes asignaturas he tenido que hacer uso de MatLab, siendo las nociones impartidas en esta asignatura de gran ayuda para programar o llevar a cabo funciones más complejas con esta herramienta. En la misma línea, en esta asignatura tuve mi primera toma de contacto con el sistema operativo Linux y su línea de comandos, usado en la mayoría de las asignaturas, especialmente las de la rama de telemática, haciendo que los conocimientos en Linux sean algo fundamental para el Grado en Ingeniería de Tecnologías de Telecomunicación y más para mi caso en concreto, ya que me encuentro estudiando la rama de Telemática del grado, donde el uso de Linux, o al menos el uso de las líneas de comandos de cualquier sistema operativo, es constante y obligatorio para la programación y configuración de los instrumentos del laboratorio de telemática: routers, switches, PABX, etc.

Por último, otros conocimientos teóricos, como tasas de transferencia, codificación de imágenes, textos, vídeos, sonidos..., incluso programación de bases de datos, han sido prácticos pues muchos de estos conceptos los he tenido que aplicar a alguna que otra asignatura.

\section{Conclusiones}

Fundamentos de Informática es una asignatura que sirve como toma de contacto para posteriores asignaturas del grado, por lo cual lo hace bastante útil a la hora de aprender conocimientos básicos y necesarios para la comprensión de otras asignaturas del grado, que no serán continuación de ésta, pero sí que en cierto modo toman como cimientos algunos conceptos aprendidos en esta asignatura. Además, recuerdo que la asignatura se me hizo amena, con unas prácticas bien planificadas (si era necesario, acompañadas de un seminario) y bien repartidas, lo que favorecía la comprensión de lo que se estaba haciendo y la finalización de las prácticas de una manera satisfactoria.

Quizás, como parte negativa de la asignatura, es que en algunos momentos se echaba en falta algo más temario de prácticas, ya que en alguna ocasión al 
enfrentarnos a los problemas, al no haber incidido mucho en ese aspecto práctico, encontraba algunas dificultades. También, en el apartado de prácticas, en el seminario sobre el montaje de un PC eché en falta que se nos enseñase de manera práctica cómo montar un PC y el por qué de cada conexión y cómo funcionaban, ya que, aunque bien es cierto que lo vimos de manera teórica, algo más práctico lo habría hecho más tangible, por lo que probablemente podría mejorar nuestro aprendizaje.

No obstante, he de decir que es una asignatura a la que guardo buen recuerdo ya que el temario y las clases que se impartían me resultaban bastante interesantes, además de que todos los contenidos estaban bastante bien explicados y se nos aportaba suficiente material para poder entender correctamente la asignatura y lograr unos buenos objetivos finales.

Agradecimientos. Agradezco a Héctor Pomares Cintas su colaboración e implicación durante el proceso de creación de este artículo para que arribase a buen puerto.

\section{Referencias}

1. Prieto, A., Lloris, A. y Torres, J. C. Introducción a la informática. Ed. McGraw- Hill, 2006.

2. Universidad de Granada. Guía docente de la asignatura Fundamentos de Informática. http://grados.ugr.es/telecomunicacion/pages/infoacademica/guias_docentes/bsicas/ fundamentos-tecnolgicos-y-empresariales/fundamentos-de-informtica/! 